\title{
Effects of notoginseng leaf triterpenes on small molecule metabolism after cerebral ischemia/reperfusion injury assessed using MALDI-MS imaging
}

\author{
Lei Wang ${ }^{1,2 \#}$, Ting Zhu ${ }^{1 \#}$, Hui-Bo Xu ${ }^{3}$, Xiao-Ping Pu${ }^{4}$, Xin Zhao ${ }^{4}$, Fang Tian ${ }^{4}$, Tao Ding ${ }^{3}$, Gui-Bo Sun ${ }^{1}$, \\ Xiao-Bo Sun ${ }^{1}$
}

${ }^{1}$ Beijing Key Laboratory of Innovative Drug Discovery of Traditional Chinese Medicine (Natural Medicine) and Translational Medicine, Institute of Medicinal Plant Development, Peking Union Medical College and Chinese Academy of Medical Sciences, Key Laboratory of Bioactive Substances and Resources Utilization of Chinese Herbal Medicine, Ministry of Education, Institute of Medicinal Plant Development, Chinese Academy of Medical Sciences \& Peking Union Medical College, Key Laboratory of New Drug Discovery based on Classic Chinese Medicine Prescription, Chinese Academy of Medical Sciences, Beijing, China; ${ }^{2}$ Harbin University of Commerce, Harbin, China; ${ }^{3}$ Jilin Academy of Chinese Medicine, Jilin, China; ${ }^{4}$ National Key Research Laboratory of Natural and Biomimetic Drugs, Peking University, Beijing, China

Contributions: (I) Conception and design: L Wang, T Zhu; (II) Administrative support: XB Sun; (III) Provision of study materials or patients: L Wang, T Zhu; (IV) Collection and assembly of data: L Wang, T Zhu, GB Sun; (V) Data analysis and interpretation: All authors; (VI) Manuscript writing: All authors; (VII) Final approval of manuscript: All authors.

\#These authors contributed equally to this work.

Correspondence to: Xiao-Bo Sun. Institute of Medicinal Plant Development, Peking Union Medical College and Chinese Academy of Medical Sciences, Beijing 100193, China. Email: sun-xiaobo@163.com; Gui-Bo Sun. Institute of Medicinal Plant Development, Peking Union Medical College and Chinese Academy of Medical Sciences, Beijing 100193, China. Email: sunguibo@126.com.

Background: Notoginseng leaf triterpenes (PNGL) is believed to have neuroprotective effects via the inhibition of inflammatory response and neuronal apoptosis. However, its mechanisms underlying the antiischemia/reperfusion (I/R) injury effects on the regulation of small molecule metabolism in rat brain remains unclear. The purpose of this study was thus to explore the mechanisms of PNGL on the regulation of small molecule metabolism in rat brain after I/R injury using matrix-assisted laser desorption ionization-mass spectrometry imaging (MALDI-MSI).

Methods: As a model of in vivo cerebral I/R injury, male Sprague-Dawley (SD) rats were established with a middle cerebral artery occlusion/reperfusion (MCAO/R) model after PNGL administration with $40 \mathrm{mg} \cdot \mathrm{kg}^{-1}$ through intraperitoneal injection (i.p.) for 7 days. We assessed the neurological behavior, regional cerebral blood flow (r CBF), neuron injury, and spatial distribution of metabolic small molecules.

Results: Our in vivo results suggested that PNGL increased cerebral blood flow and relieved neurological dysfunction. Furthermore, using MALDI-MSI, we demonstrated that PNGL regulated 16 endogenous small molecules implicated in metabolic networks including tricarboxylic acid (TCA) cycle, adenosine triphosphate (ATP) metabolism, malate-aspartate shuttle, metal ions, and antioxidants underwent noticeable changes after reperfusion for $24 \mathrm{~h}$.

Conclusions: PNGL is a novel cerebrovascular agent that can improve cerebral blood flow and attenuate adverse neurological disorders. The mechanisms are closely correlated with relative metabolic pathways, which offers insight into exploring new mechanisms in PNGL for the treatment of cerebral I/R injury.

Keywords: Cerebral I/R injury; notoginseng leaf triterpenes (PNGL); matrix-assisted laser desorption ionizationmass spectrometry imaging (MALDI-MSI); small molecule metabolism

Submitted Jun 23, 2020. Accepted for publication Nov 19, 2020.

doi: 10.21037/atm-20-4898

View this article at: http://dx.doi.org/10.21037/atm-20-4898 


\section{Introduction}

Stroke is a complex disease that affects different brain regions, leading to disruption of abundant neural circuits, and it involves complicated injury cascade responses (1). Of all stroke events, ischemic stroke (caused by decreased blood flow to the brain) accounts for approximately $87 \%$ in nature (2). Ischemic stroke causes a complex pathophysiological response including destruction of the neurovascular unit, neuronal death and inflammation $(3,4)$. Rapid supply of cerebral blood flow is the primary target of ischemic stroke treatment and the prerequisite for neuroprotective therapies (5). Except for tissue plasminogen activator (tPA), few pharmacological agents have succeeded in clinical trials. Therefore, the search for promising neuroprotective compounds to restore cerebral blood flow and improve neurological deficits after ischemic stroke to overcome the clinical therapeutic limitations remains an urgent issue.

Currently, matrix-assisted laser desorption/ionization mass spectrometry imaging (MALDI-MSI) is an innovative methodology that enables label-free localization of molecular species in tissue sections mounted on electrically conductive slides (6). By means of unique advantages of label free, molecular specificity, high-throughput analysis, simple sample preparation, and in situ positioning (7), MALDI-MSI plays an irreplaceable role for measuring the spatial distribution of small molecules $(8,9)$, metabolites (10), proteins (11), peptides (12), lipids (13), or drugs (14). Furthermore, recent improvements in MALDI-MSI have been shown to be useful for different kinds of cerebrovascular disease $(8,15)$.

Panax notoginseng, is a traditional Chinese medicinal plant that has a long history of use in China for preventing and treating ischemic cerebrovascular diseases (16). The current research on Panax notoginseng mainly focuses on the roots of Panax notoginseng. However, the stem and leaf parts of Panax notoginseng are often ignored. Notoginseng leaf triterpenes (PNGL) is the total saponins extracted from the stem and leaf of Panax notoginseng, which has the functions of regulating blood lipid, sedative and hypnotic, analgesic, anti-inflammatory, and anti-aging (17-20). More importantly, our previous studies have mainly reported the neuroprotective effect of PNGL after ischemic stroke in the inhibition of inflammation and apoptosis (21). However, the mechanism of PNGL on brain metabolic small molecules and their spatial distributions remain unsystematic explored.

The present study is the first to investigate the protective effects of PNGL on cerebral I/R injury correlated with cerebral blood flow, neurological scores, and histomorphology. MALDI-MSI was used to further explore the anticerebral I/R injury effects of PNGL on the metabolism of endogenous substances in relation to glucose metabolism, ATP metabolism, antioxidant molecules, and metal ions. Additionally, chemical fingerprinting data have revealed that the content of total saponins of Panax notoginseng stems and leaves are mainly protopanaxadiol-type saponins, and contains almost no protopanaxatriol-type saponins (21). As shown in Figure S1A,B, monomeric saponins contained in protopanaxadiol-type saponins mainly include ginsenoside $\mathrm{Rb} 1$, ginsenoside $\mathrm{Rc}$, ginsenoside $\mathrm{Rb} 2$, and ginsenoside $\mathrm{Rb} 3$. In addition, eleven batches of PNGL samples were detected by the fingerprinting assay (Figure S2).

We present the following article in accordance with the ARRIVE reporting checklist (available at http://dx.doi. org/10.21037/atm-20-4898).

\section{Methods}

\section{Drugs}

PNGL was supplied by Jilin Academy of Chinese Medicine (Jilin, China). A positive drug dl-3-n-butylphthalide (NBP) was purchased from CSPC-NBP Pharmaceutical Co., Ltd (Hebei, China). Before study, they were freshly dissolved in $0.9 \%$ normal saline.

\section{Animals}

Male Sprague-Dawley (SD) rats (purchased from Beijing Vital River Laboratories, Beijing, China) weighing 250$280 \mathrm{~g}$ were used in this study. The study was approved by the Institutional Animal Care and Use Committee of Chinese Academy of Medical Sciences and Peking Union Medical College (SYXK 2017-0020). All rats care and experimental procedures were reported in accordance with the Laboratory Animal Ethics Committee of the Institute of Medicinal Plant Development, Peking Union Medical College and complied with NIH Guidelines for the Care and Use of Laboratory Animals. All rats were randomized into four experimental groups, as followed: sham group, $\mathrm{MCAO} / \mathrm{R}$ model group, PNGL (40 $\mathrm{mg} \cdot \mathrm{kg}^{-1}$ ) group, and NBP (20 mg. $\left.\mathrm{kg}^{-1}\right)$ group. For drug administration, PNGL, NBP or $0.9 \%$ normal saline was exposed to continuous intraperitoneal injection (once per day) for $7 \mathrm{~d}$ prior to MCAO surgery. At $24 \mathrm{~h}$ after reperfusion, some brain tissues were fixed for histopathology and small molecule 
metabolism detection.

\section{Middle cerebral artery occlusion/reperfusion (MCAO/ R) surgery and regional cerebral blood flow (r CBF) measurement}

Male SD rats were anaesthetized with ketamine $\left(80 \mathrm{mg} \cdot \mathrm{kg}^{-1}\right)$ and xylazine $\left(10 \mathrm{mg} \cdot \mathrm{kg}^{-1}\right)$ via intraperitoneal injections. $\mathrm{MCAO} / \mathrm{R}$ was proceeded by experimenters who were unaware of the grouping scheme, following a previously described method (22). After MCAO surgery, the wound was disinfected with iodine, and then the wound was sutured with sterile surgical suture to reduce the bleeding. We also injected tramadol $\left(2.5 \mathrm{mg} \cdot \mathrm{kg}^{-1}\right)$ by tail intravenous to relieve the pain caused by the operation. Reperfusion was induced for $24 \mathrm{~h}$ by removing the suture. Rats in the sham group were operated using the same method but with no occlusion in middle cerebral artery. The body temperature was maintained at $37 \pm 0.5^{\circ} \mathrm{C}$ until rats woke up using a heating pad (Sunbeam, USA). The incision was sewed after ischemia and cerebral blood flow in the ischemic core region and peripheral region after reperfusion $24 \mathrm{~h}$ was assessed by the laser Doppler flowmeter (moor FLPI-2, UK).

\section{Neurological score}

Neurological performances of all animals were performed by two blinded investigators using a 5 -point scale following a previously described method (22). The neurological function was scored according to a series of scales from 0 to 4 . The highest score represents the most severe neurological deficits.

\section{Histopathology staining}

Histopathology staining ( $24 \mathrm{~h}$ postreperfusion) was implemented as reported previously (23). The brain samples were embedded in paraffin and coronally dissected into 5 - $\mu$ m-thick sections. Then paraffin sections were stained with hematoxylin-eosin (H\&E) and Nissl staining to reveal histopathological lesions.

\section{MSI}

Frozen sections $(10 \mu \mathrm{m})$ of the brain tissues were taken for MALDI-MSI using a Leica CM1950 cryostat (Leica Microsystems GmbH, Wetzlar, Germany) $-20^{\circ} \mathrm{C}$ and placed the thawed brain tissue slices on electrically conductive glass slides coated with indium tin oxide (ITO). The MALDI
MSI experiments were implemented using the Autoflex SpeedTM MALDI-TOF/TOF MS (Bruker Daltonics, Bremen, Germany) as described reported previously $(8,24)$.

\section{Data and statistical analysis}

Experimental data are expressed as the means \pm standard deviation (SD) and were statistically evaluated using SPSS17 software (IBM Corporation, New York, NY, USA). Differences between the groups were analyzed by one-way ANOVA and Tukey's post hoc test ( $t$-test), and $\mathrm{P}<0.05$ was considered statistically significant.

\section{Results}

\section{PNGL increased $r$ CBF and reduced neurologic deficits}

As shown in Figure $1 A, B$, the $\mathrm{r}$ CBF in different groups was evaluated using a laser Doppler flowmeter system. The results revealed that the administration of PNGL and NBP for 7 days resulted in a significant increase in $\mathrm{r} C B F$ at $24 \mathrm{~h}$ after reperfusion versus the model group. Meanwhile, a visible amelioration in neurologic deficit was observed in PNGL administration for 7 days (Figure 1C). In addition, NBP did not exhibit significant defferences on the neurological deficit scores compared with the PNGL.

\section{PNGL inbibited neuronal loss}

As shown in Figure 1D,E, H\&E-stained slides of brain sections from each group were detected under a light microscope. Untreated rats have many neurons present in the pyknotic nuclei and weak staining, whereas MCAO/ $\mathrm{R}$ rats have pale nuclei in the cerebral cortex regions. In contrast, administration of PNGL for 7 days prior to MCAO notably reduced the pyknotic nuclei in the cerebral cortex. Analogously, as shown in Figure 1F, G, Nissl staining from each group were also detected under a light microscope. $\mathrm{MCAO} / \mathrm{R}$ rats have many neurons present in the shrunken phenotype and weak staining in the cerebral cortex. However, compared with the model group, PNGL administration for 7 days prior to MCAO exhibited strong staining in the cerebral cortex. Additionally, NBP did not exhibit significant differences on neuronal loss compared with the PNGL.

\section{PNGL decreased the interruption of TCA cycle}

The results of Figure 2A,B demonstrated that the MCAO/ 
A
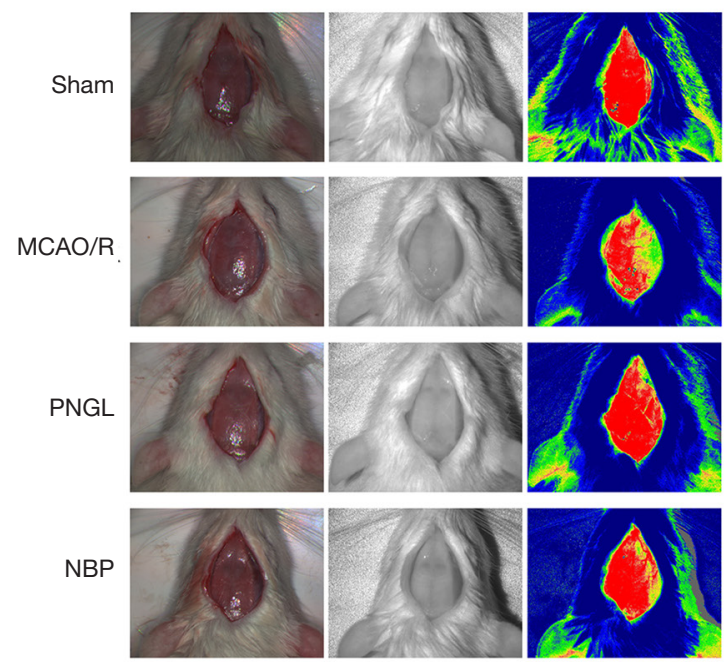

D
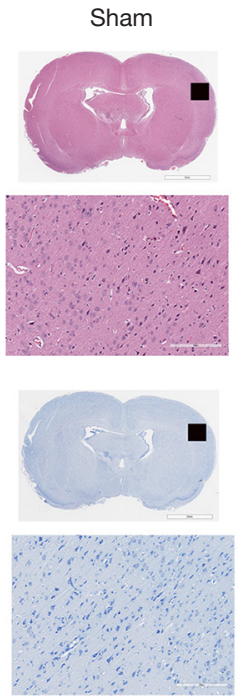

$\mathrm{MCAO} / \mathrm{R}$
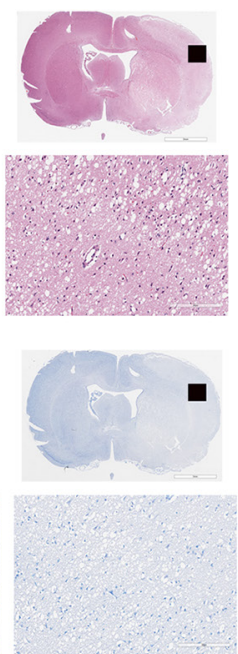

PNGL
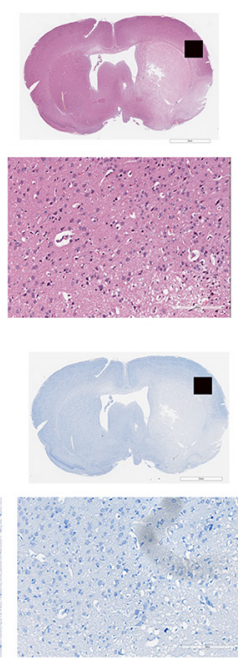

NBP
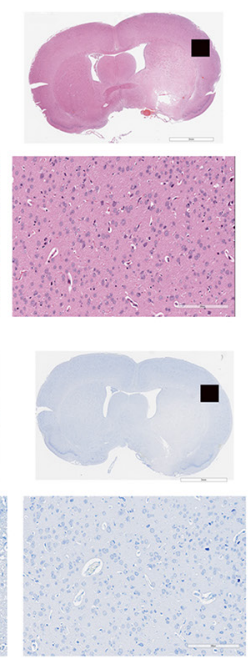

B

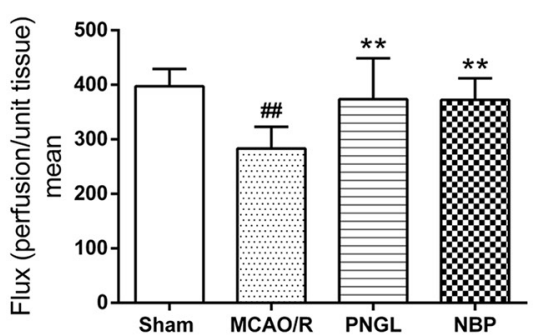

C

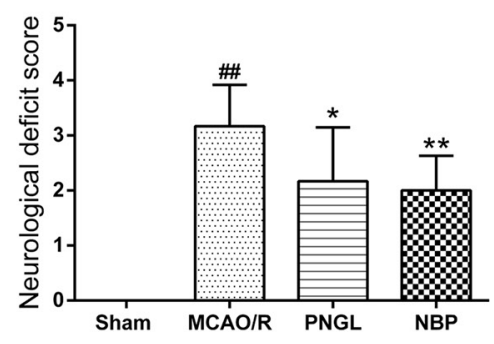

E

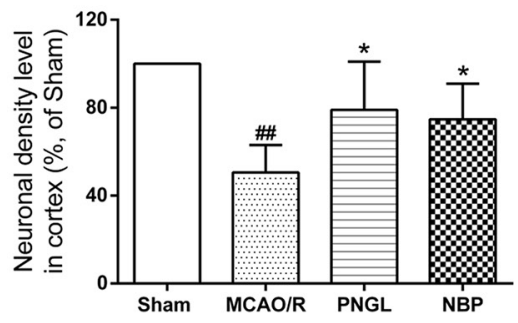

$\mathrm{F}$

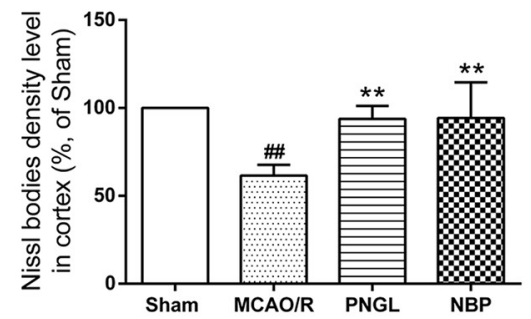

Figure 1 PNGL improves $r$ CBF and decreases neuronal loss. (A) The images of $r$ CBF in the cortex region. The magnitude of $r$ CBF is expressed by different colors, and blue represents the most severe ischemia. (B) Flux mean value of $r$ CBF in different groups, $n=5-6$ per group. (C) Neurological deficit scores are assessed in rats with MCAO $2 \mathrm{~h}$ then reperfusion $24 \mathrm{~h}, \mathrm{n}=6$ per group. (D) Pathological sections stained with $\mathrm{H} \& \mathrm{E}$ in the cortex region for each group. (E) Quantitative analysis in the cortex region in all groups of $\mathrm{H} \& \mathrm{E}$ staining, $\mathrm{n}=4$ rats per group, scale bar $=200 \mu \mathrm{m}$. (F) Pathological sections stained with Nissl in the cortex region for each group. (G) Quantitative analysis in the cortex region in all groups of Nissl staining, $\mathrm{n}=4$ rats per group, scale bar $=200 \mu \mathrm{m}$. Data are expressed as the mean $\pm \mathrm{SD}$. ${ }^{\#} \mathrm{P}<0.01$ vs. sham group; ${ }^{*} \mathrm{P}<0.05,{ }^{*} \mathrm{P}<0.01$ vs. $\mathrm{MCAO} / \mathrm{R}$ group. $\mathrm{PNGL}$, notoginseng leaf triterpenes; r CBF, regional cerebral blood flow; MCAO, middle cerebral artery occlusion; $\mathrm{H} \& \mathrm{E}$, hematoxylin-eosin.

$\mathrm{R}$ model group remarkably increased the content of glucose (Figure $2 A$ ) and citric acid (Figure $2 B$ ) in rats. However, PNGL can significantly decreased the content of glucose and citric acid in both the striatum and cerebral cortex. Moreover, NBP did not exhibit significant differences on the content of glucose and citric acid compared with the PNGL.

\section{PNGL increased the glutamine content and improved the malate-aspartate shuttle}

MALDI-MSI maps of glutamine, aspartate and 


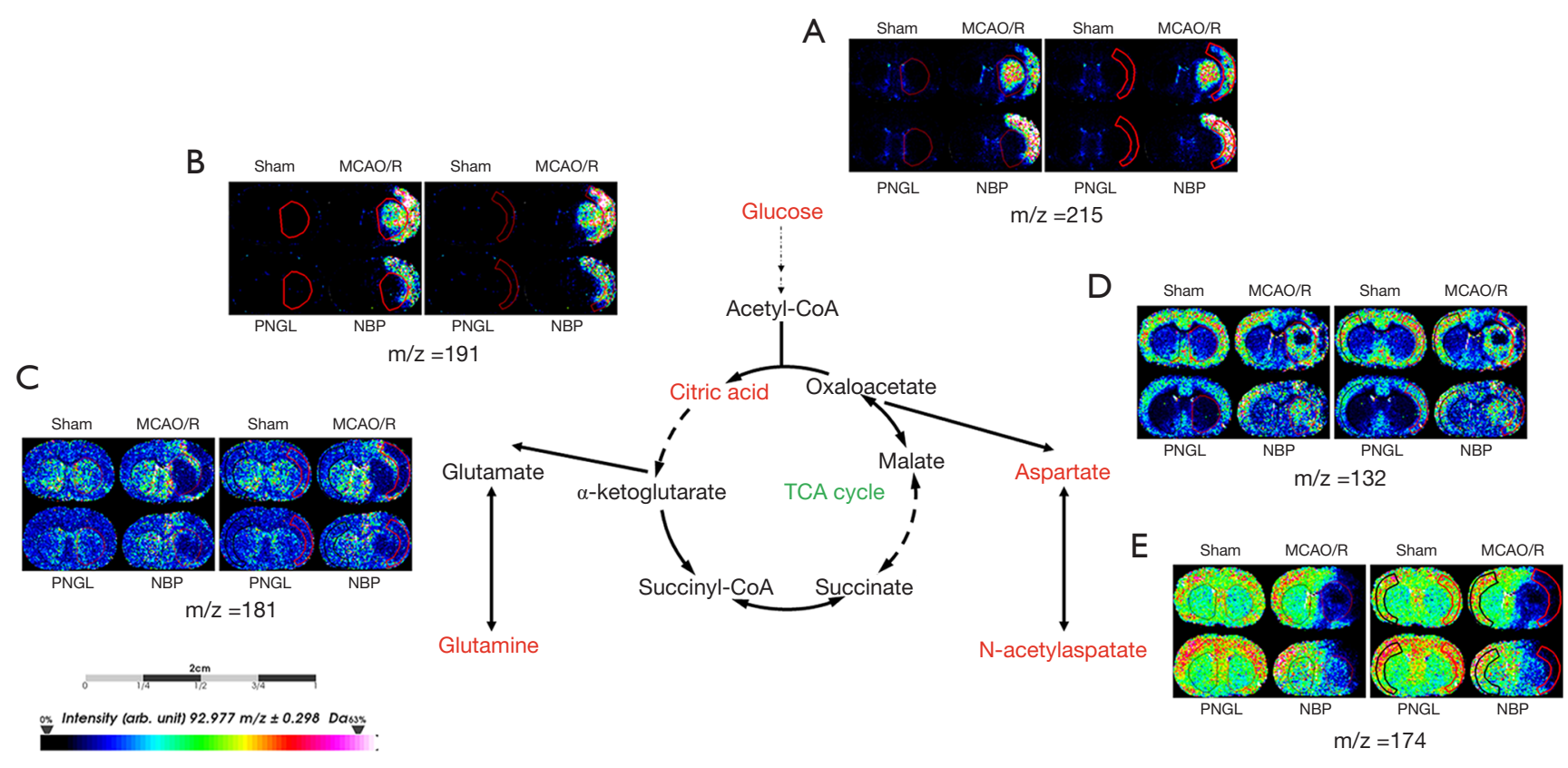

$\mathrm{F}$

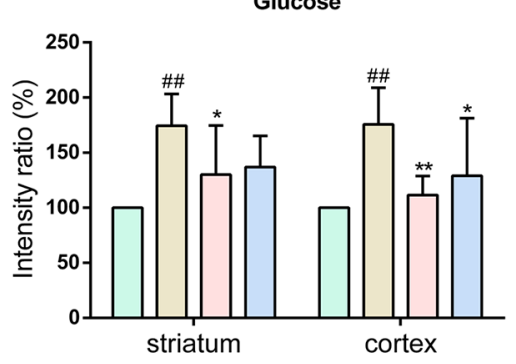

Aspartate

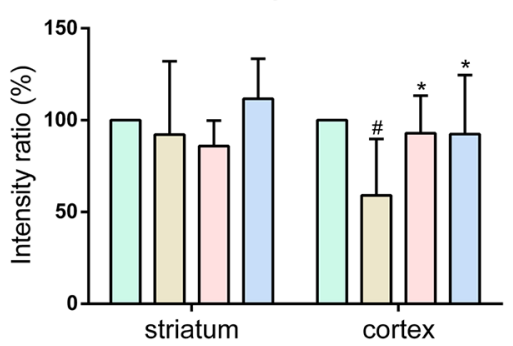

Citric acid

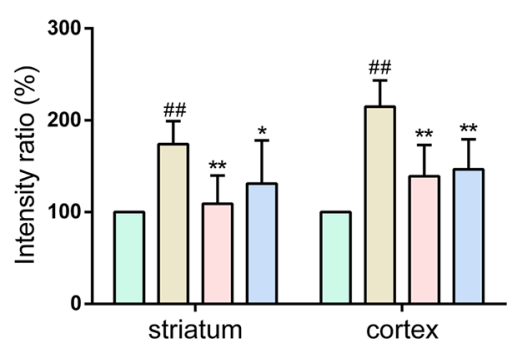

N-acetyl-L-Aspartate
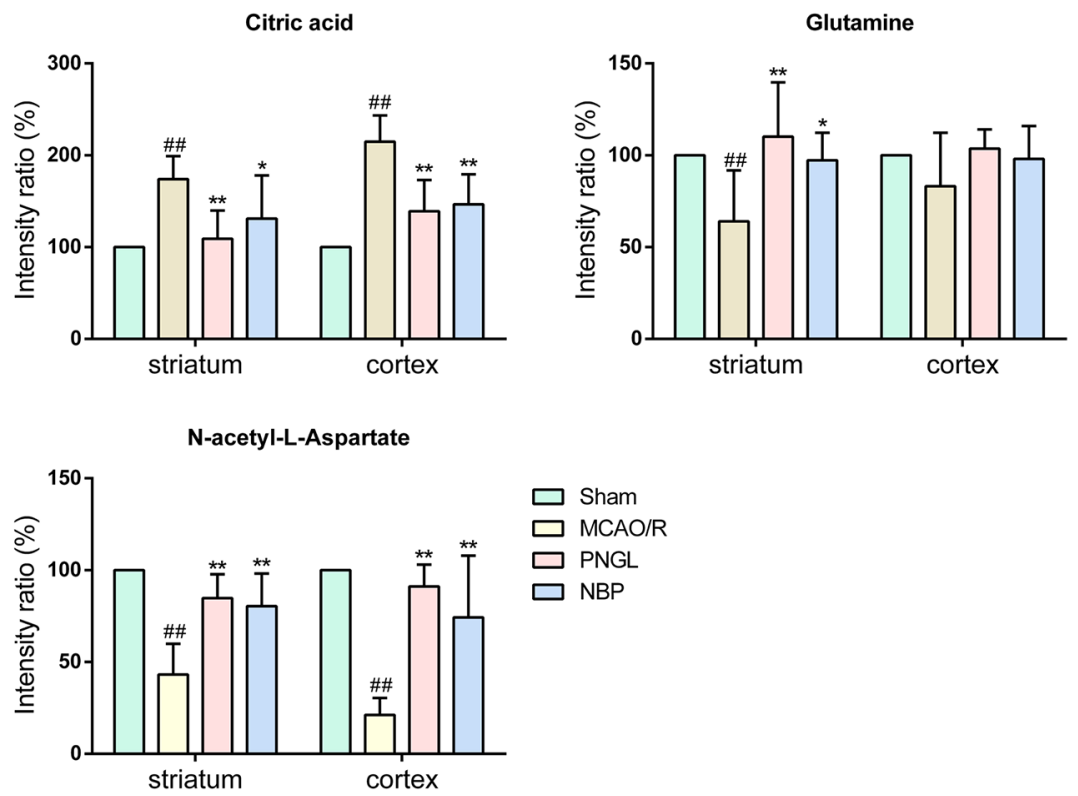

Figure 2 PNGL decreases the interruption of TCA cycle and improves the malate-aspartate shuttle. Ion image distributions of (A) glucose, (B) citric acid, (C) glutamine, (D) aspartate, and (E) N-acetylaspartate. (F) Quantitative data of glucose, citric acid, glutamine, aspartate and $\mathrm{N}$-acetylaspartate in the striatum and cerebral cortex. Data are expressed as the mean $\pm \mathrm{SD}(\mathrm{n}=5)$, scale bar $=2 \mathrm{~cm} .{ }^{\prime \prime} \mathrm{P}<0.01$ vs. sham group; ${ }^{*} \mathrm{P}<0.05,{ }^{* *} \mathrm{P}<0.01$ vs. MCAO/R group. $\mathrm{PNGL}$, notoginseng leaf triterpenes; $\mathrm{MCAO} / \mathrm{R}$, middle cerebral artery occlusion/reperfusion; MALDI MSI, matrix-assisted laser desorption ionization-mass spectrometry imaging. 
$\mathrm{N}$-acetylaspartate (NAA) were observed as shown in Figure $2 C, D, E$, respectively. The results revealed that the MCAO/ $\mathrm{R}$ model group decreased the content of glutamine in the striatum region. However, the content of glutamine was increased after PNGL administration (Figure 2C). Our results also revealed that the aspartate content decreased in the ischemic region while PNGL obviously increased the aspartate level in the cerebral cortex (Figure 2D). The distribution of NAA shown in Figure $2 E$ demonstrated that the content of NAA decreased in the ischemic region. PNGL administration for 7 days increased the level of NAA both in the striatum and cerebral cortex. However, the other regions in rat brain were not significantly changes in aspartate. In addition, NBP did not exhibit significant differences on the changes of glutamine, aspartate and NAA compared with the PNGL.

\section{PNGL increased ATP metabolism}

As shown in Figure 3, the MCAO/R rats showed low levels of GMP (A), AMP (B) and ADP (C) and the consequently decreased content of other related substances including adenosine (D) and inosine (E) in the ischemic region, whereas the $\mathrm{MCAO} / \mathrm{R}$ rats showed high levels in the infarct area. However, we found that PNGL administration significantly increased the levels of AMP, ADP, adenosine, and inosine in both the striatum and cerebral cortex. The level of GMP in the cerebral cortex was relatively higher than that in the striatum. In addition, PNGL administration for 7 days can significantly decrease xanthine content in both the striatum and cerebral cortex region. Moreover, NBP did not exhibit significant differences on the changes of ATP-related metabolites compared with the PNGL.

\section{PNGL increased the content of antioxidants}

As shown in Figure 4A, B, C, MCAO/R model group decreased the levels of glutathione (A), ascorbic acid (AA) (B) and taurine $(\mathrm{C})$ in the striatum and cerebral cortex regions. However, the amount of glutathione, AA and taurine were significantly increased in both the striatum and cerebral cortex after PNGL administration. Moreover, NBP did not exhibit significant differences on the content of antioxidants compared with the PNGL.

\section{PNGL maintained the homeostasis of $\mathrm{Na}+$ and $\mathrm{K}+$}

We also evaluated the content of $\mathrm{Na}+$ and $\mathrm{K}+$ using 1 ,
5-DAN hydrochloride as a matrix. Figure 4 showed that $\mathrm{MCAO} / \mathrm{R}$ model group increased $\mathrm{Na}+$ level, whereas the level of $\mathrm{K}+$ remarkably decreased both in the striatum and cerebral cortex regions compared with the sham group. However, PNGL administration for 7 days significantly reduced the $\mathrm{Na}+$ level and increased $\mathrm{K}+$ level.

\section{Discussion}

Insufficient supply of oxygen and glucose caused by middle cerebral artery occlusion causes sodium-potassium pump imbalance and leads to membrane depolarization, which is mainly reflected in the destruction of TCA cycle and the blocking of ATP synthesis and metabolism (7). Finally, neuronal death occurred due to excitotoxicity, mitochondrial dysfunction, immune response, and acidotoxicity caused by membrane depolarization (5).

PNGL is the total saponins extracted from the stems and leaves of Panax notoginseng. Many reports have demonstrated that PNGL exerts a neuroprotective effect in alleviating neurological disorders through the suppression of inflammation, apoptosis and autophagy $(21,25)$. PNGL was found to counteract aberrant autophagy and apoptosis in hippocampal neurons of mice with cognitive impairment induced by sleep deprivation (25). Importantly, our previous study has shown that the neuroprotective effects of PNGL against cerebral I/R injury via inhibiting inflammatory response and neuronal apoptosis (21). In this work, we found that PNGL had a neuroprotective effect on cerebral I/R injury by improving the cerebral blood flow, neurological function, and the levels of small molecule metabolism during the acute phase after ischemic stroke.

Glucose is the most important and basic energysupplying substance of various organisms, and the major energy source in the human brain and nervous system. Citric acid is the main mediator of the TCA cycle, and it has been reported to be concentrated in the striatum and cerebral cortex of ischemic areas (7). Our data showed that the contents of glucose and citric acid had significant increase in the right cerebral cortex and striatum compared with the sham group. Importantly, PNGL administration can significantly decreased this abnormal accumulation of glucose and citric acid in both the striatum and cerebral cortex.

Glutamine is mainly located in astrocytes and is essential for maintaining nitrogen homeostasis and ammonia detoxification in the brain. In addition, glutamine can also ensure the effective circulation of the neurotransmitters 
A

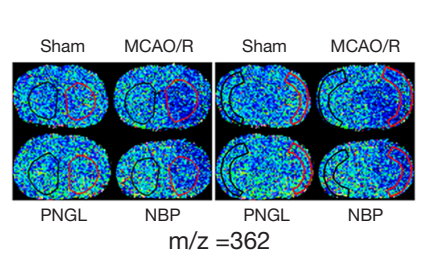

B
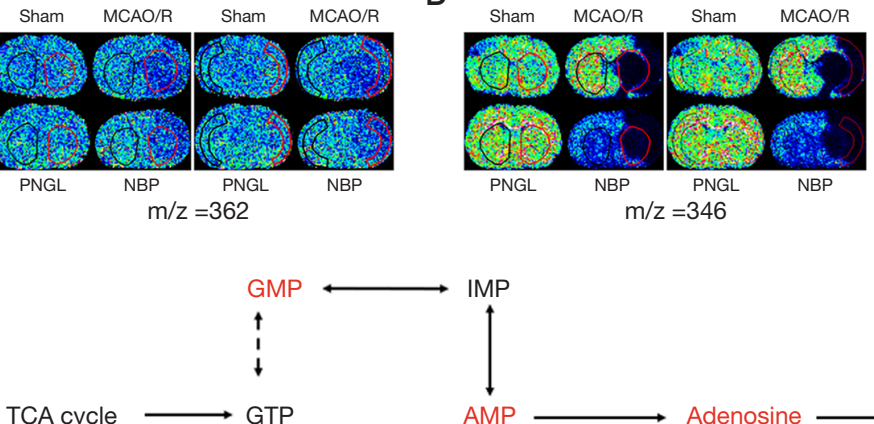

TCA cycle

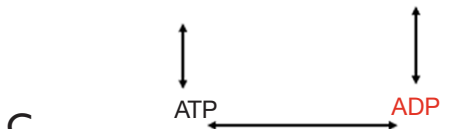

C

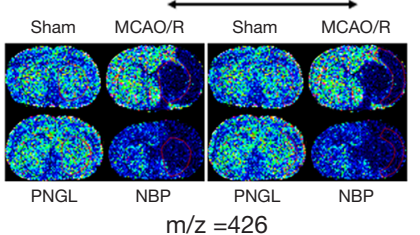

$0 \quad \frac{2 \mathrm{~cm}}{\sqrt{4} / 2}$

ox Intensity (arb. unit) $92.977 \mathrm{~m} / \mathrm{z} \pm 0.298$ Dasus

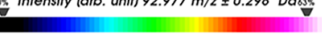

G

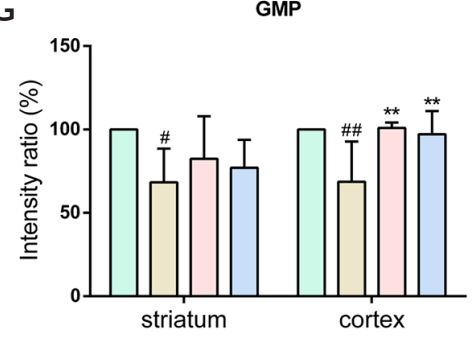

Adenosine
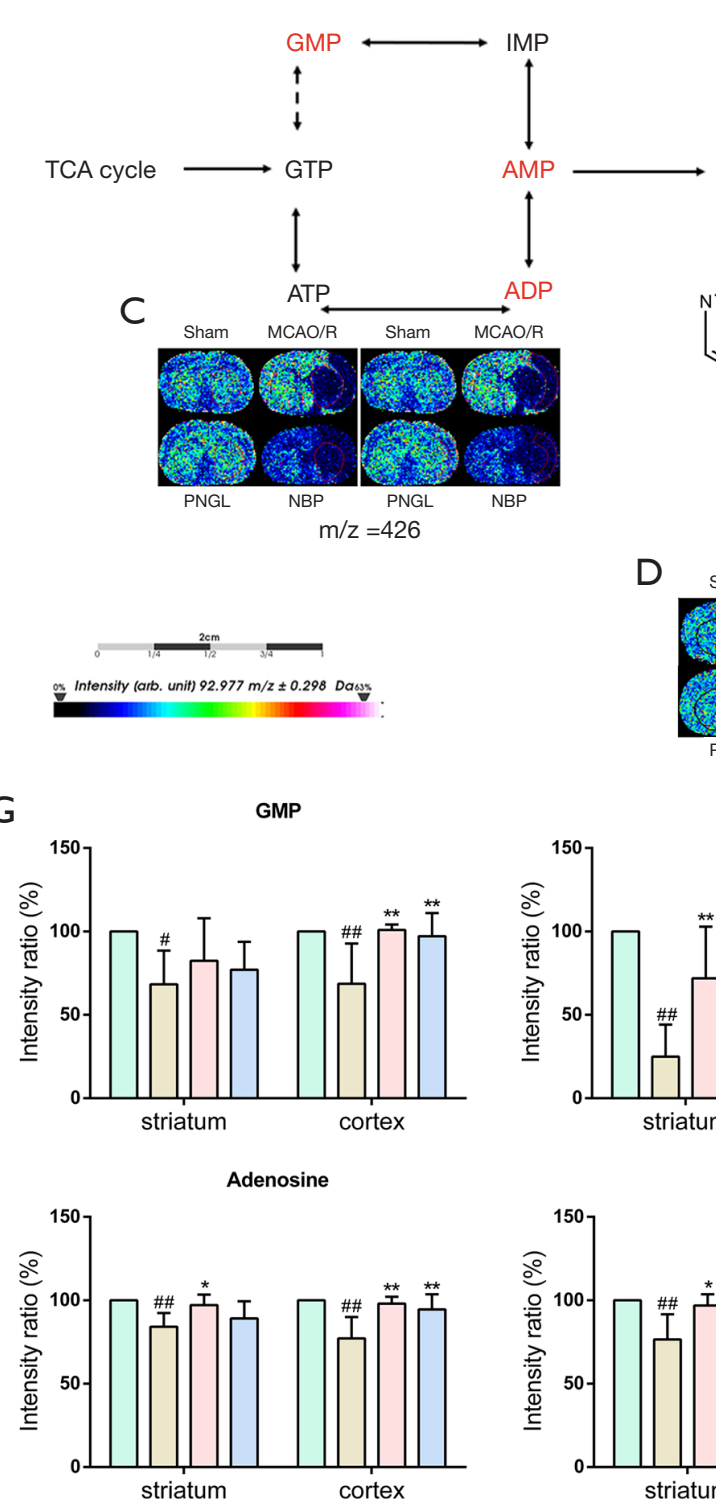

Adenosine

D

$\mathrm{m} / \mathrm{z}=302$

AMP
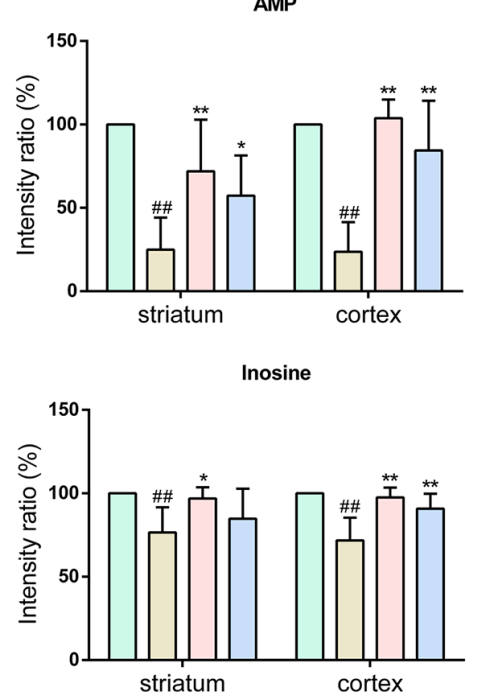

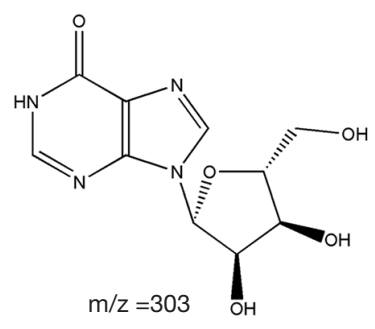

E
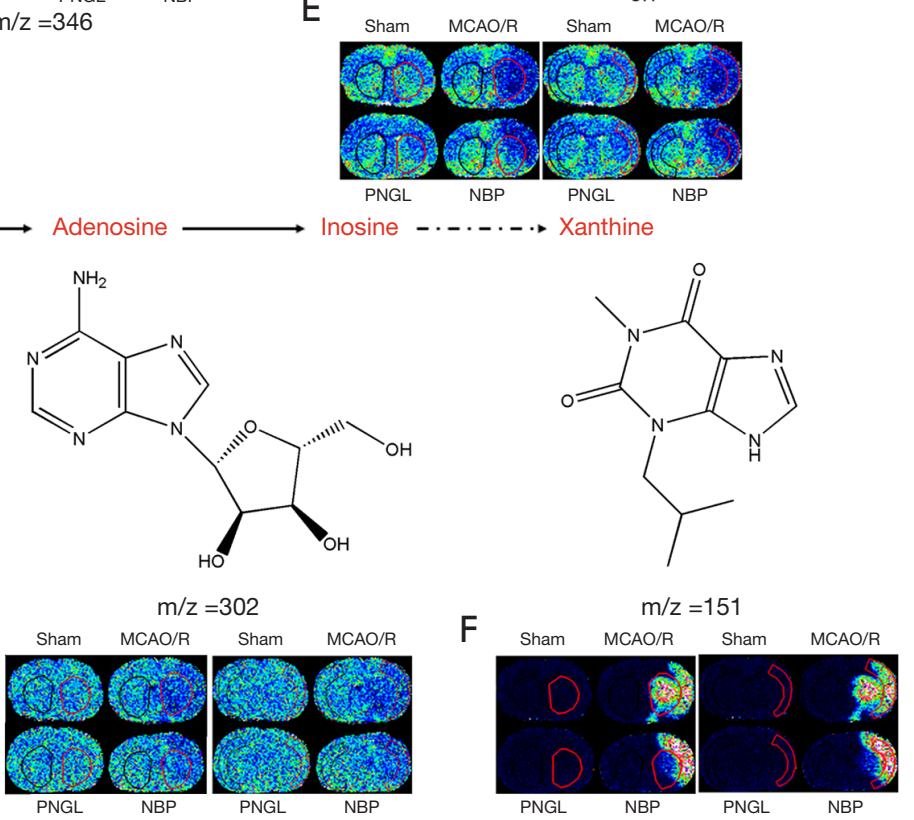

ADP
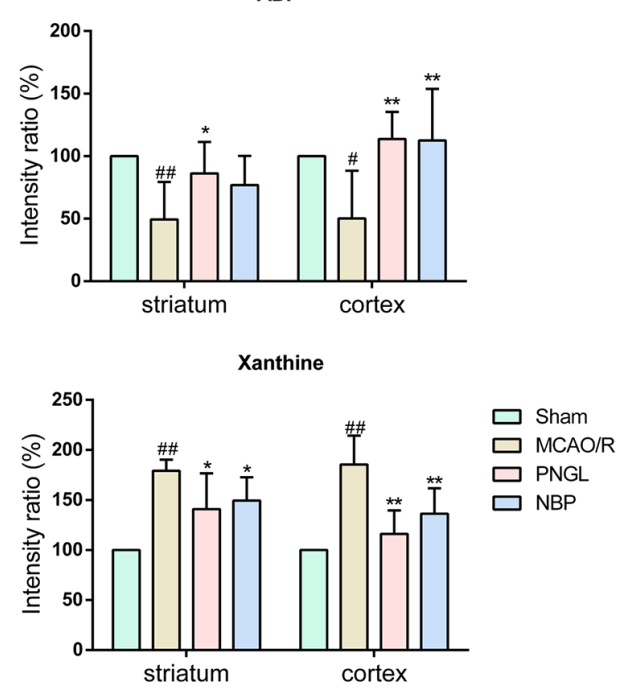

Figure 3 PNGL increases ATP metabolism. Ion image distributions of (A) GMP, (B) AMP, (C) ADP, (D) adenosine, (E) inosine, (F) xanthine. (G) Quantitative data of GMP, AMP, ADP, adenosine, inosine and xanthine in the striatum and cerebral cortex. Data are expressed as the mean $\pm \mathrm{SD}(\mathrm{n}=5)$, scale bar $=2 \mathrm{~cm} .{ }^{*} \mathrm{P}<0.05,{ }^{\# \#} \mathrm{P}<0.01$ vs. sham group; ${ }^{*} \mathrm{P}<0.05,{ }^{*} \mathrm{P}<0.01$ vs. MCAO/R group. PNGL, notoginseng leaf triterpenes; $\mathrm{MCAO} / \mathrm{R}$, middle cerebral artery occlusion/reperfusion; MALDI MSI, matrix-assisted laser desorption ionization-mass spectrometry imaging; ATP, adenosine triphosphate; GMP, guanosine monophosphate; AMP, adenosine monophosphate; ADP, adenosine diphosphate. 
A

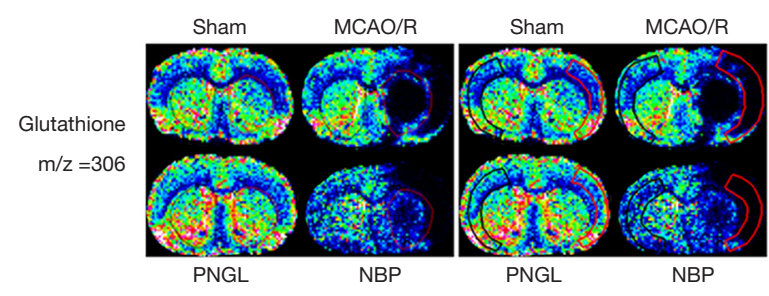

B

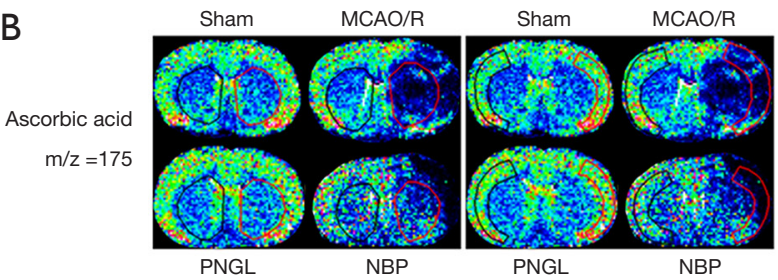

C

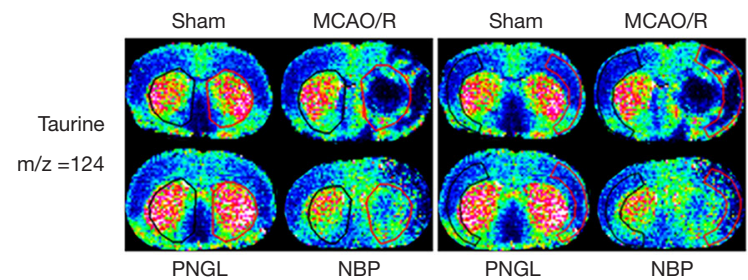

D

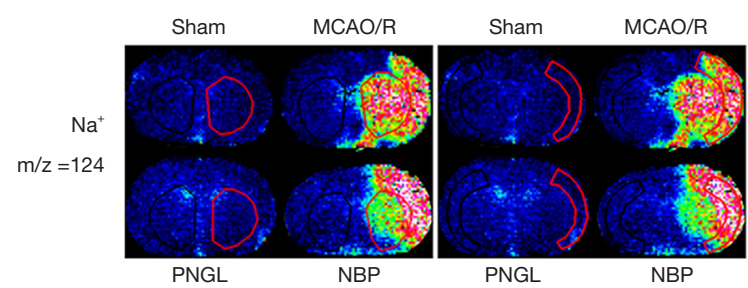

$\mathrm{E}$

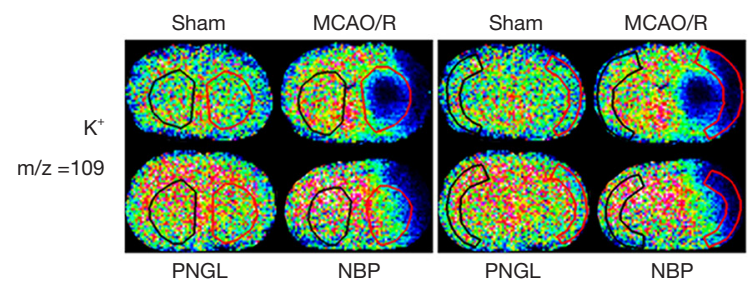

$\mathrm{F}$
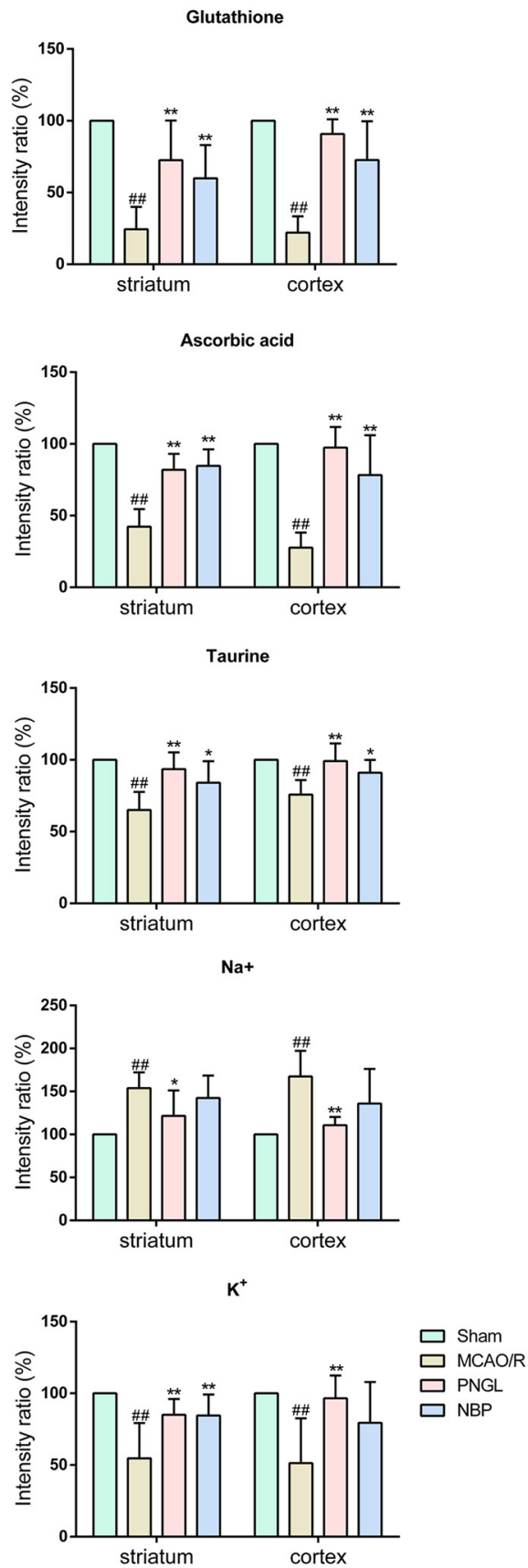

Figure 4 PNGL increases the content of antioxidants and maintains the homeostasis of $\mathrm{Na}^{+}$and $\mathrm{K}^{+}$. Ion image distributions of (A) glutathione, (B) ascorbic acid, (C) taurine, (D) $\mathrm{Na}^{+}$, (E) $\mathrm{K}^{+}$. (F) Quantitative data of glutathione, ascorbic acid, taurine, $\mathrm{Na}^{+}$and $\mathrm{K}^{+}$in the striatum and cerebral cortex. Data are expressed as the mean $\pm \mathrm{SD}(\mathrm{n}=5)$, scale bar $=2 \mathrm{~cm}$. ${ }^{* \#} \mathrm{P}<0.01$ vs. sham group; ${ }^{*} \mathrm{P}<0.05,{ }^{* *} \mathrm{P}<0.01$ vs. MCAO/R group. PNGL, notoginseng leaf triterpenes; MCAO/R, middle cerebral artery occlusion/reperfusion; MALDI MSI, matrixassisted laser desorption ionization-mass spectrometry imaging. 
between neurons and astrocytes (26). Our results showed that the content of glutamine had significant reduction in the ischemic area, whereas the content of glutamine clearly increased after PNGL administration, especially in the striatum. In addition, L-aspartate (L-Asp) exchanges glutamate and protons through mitochondrial aspartic acid/ glutamate carriers and plays an important role in the malateaspartate shuttle (27). NAA has a higher concentration in normal brain tissue, but that was significantly reduced under pathological conditions such as neuronal damage (28). Our results also showed that the contents of aspartate and NAA decreased in the ischemic hemisphere. However, PNGL administration significantly increased the contents of aspartate and NAA.

Mitochondria generates ATP by oxidative phosphorylation to become a powerhouse for eukaryotic cells (29). ATP can be catabolized to ADP, AMP, adenosine, inosine, hypoxanthine, xanthine, and eventually to uric acid following two catabolic routes known as the "IMP pathway" and the "adenosine pathway" (30). The decreased cerebral blood flow restricted oxygen delivery in the MCAO/R-treated brain, which interrupted mitochondrial oxidative phosphorylation, resulting in metabolic disturbances of ATP synthesis (24). Previous studies have reported that levels of ATP, AMP, $\mathrm{ADP}$, adenosine, hypoxanthine, and creatine were changed after MCAO challenge (8,31). In our study, we visualized the content changes and spatial distribution of 6 metabolites involved in ATP metabolism in MCAO/R-treated rats by MALDI-MSI, as presented in Figure 3. The MSI results also showed that PNGL obviously increased the ADP, GMP, AMP, adenosine, and inosine levels and consequently decreased the xanthine content in the ischemic hemisphere, suggesting that the disturbance of ATP catabolism was reversed by PNGL (7). These results indicated that the distribution and content of energy-related metabolites are affected by ischemia, mainly manifesting in the striatum and cerebral cortex.

ROS are mainly generated in the ischemic penumbra particularly after reperfusion (32), which triggers inflammatory responses, consequently increasing blood brain barrier (BBB) permeability and resulting in neuronal death (33). Glutathione (GSH), AA and taurine have the function of scavenging ROS and are considered to be an important antioxidants in the brain (15). As shown in Figure $4 A, B, C$, the content of GSH, AA, and taurine were remarkably declined in the ischemic regions, especially in the striatum and cerebral cortex. However, administration with PNGL increased the level of GSH, AA and taurine both in the striatum and cerebral cortex.

The membrane potential necessary for neuronal activity depends on the formation of normal $\mathrm{Na}^{+}$and $\mathrm{K}^{+}$concentration gradients inside and outside the cell membrane. Focal ischemia leads to abnormal mitochondrial function, which induces ATP depletion and ultimately inhibits $\mathrm{Na}^{+}-\mathrm{K}^{+}$-ATPase (34). Previous research has shown that neuronal necrosis after permanent occlusion of the middle cerebral artery are mainly due to the destruction of ion influx and efflux caused by the abnormal function of $\mathrm{Na}^{+} / \mathrm{K}^{+}$-ATPase $(35,36)$. In our research, Figure $4 D, E$ shows $\mathrm{MCAO} / \mathrm{R}$ injury increased the content of $\mathrm{Na}^{+}$, and reduced the content of $\mathrm{K}^{+}$in the striatum and cerebral cortex. These results are consistent with previous research reports, indicating that ion homeostasis was disturbed under ischemic conditions (7). Furthermore, PNGL increased the $\mathrm{K}^{+}$content and decreased the $\mathrm{Na}^{+}$content. One of the mechaniasms is that PNGL restores the dysfunction of $\mathrm{Na}^{+}-$ $\mathrm{K}^{+}$-ATPase, which normally maintains the ion influx and efflux, and finally improves neuronal necrosis after cerebral artery occlusion.

Our results also demonstrated that brain regionspecific differences are affected by PNGL in neurological function between small molecule metabolism isolated from the striatum and cerebral cortex of MCAO/R-treated rats. Thus, small molecule metabolism from the two brain regions can be regulated by different mechanisms and are therefore also likely to be differently affected by PNGL or ischemia reperfusion injury. We also showed functional differences between striatal and cortical small molecule metabolism. Regarding both ATP metabolism and antioxidants, the striatal content exhibited no difference compared with the cortical content. Meanwhile, the two brain regions compared here differed in glutamine and aspartate. Specifically, glutamine is distributed in the striatum region, and aspartate is distributed in the cerebral cortex. The obvious differences are most likely relevant to the differences in physiological function between the regions. The presented differences stress the importance of studying small molecule metabolisms from different brain regions separately, especially when exploring ischemic diseases that affect the striatum and/or the cerebral cortex.

In summary, using MALDI-MSI with assistance of 1,5-diaminonaphthalene hydrochloride to visualize small molecule metabolite systems will improve our understanding of the content and unique distribution in which small molecule metabolite act during cerebral ischemia. Figure 5 shows the effects of PNGL on 16 altered 


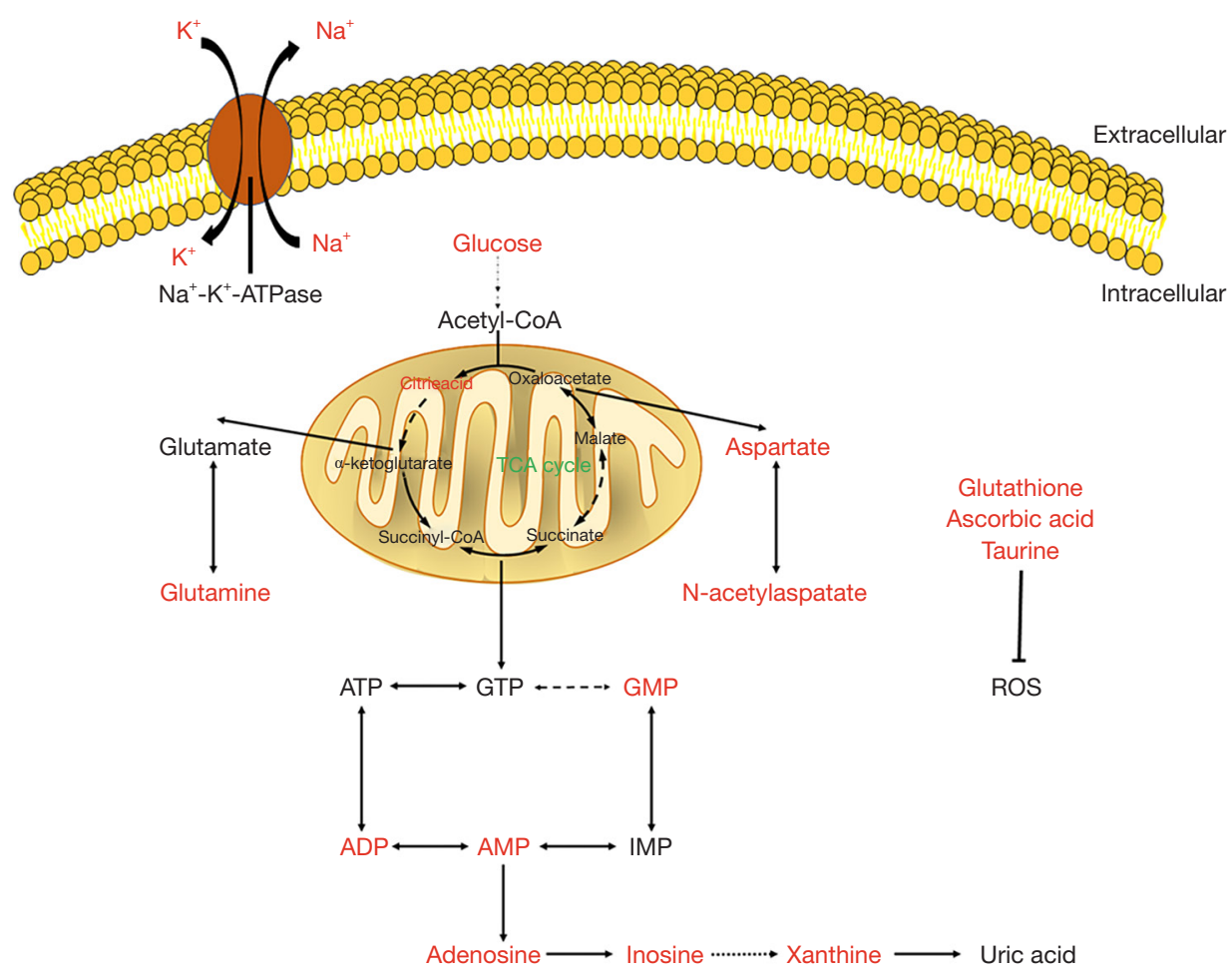

Figure 5 PNGL exerts a neuroprotective effect by regulating the abnormal small molecule metabolism in the early acute phase with MCAO rats, which is specifically reflected in the reduced abnormal accumulation of glucose and citric acid, increased ATP metabolism rate, augmented rate of the malate aspartate shuttle, increased antioxidants and maintained ionic homeostasis. PNGL, notoginseng leaf triterpenes; MCAO, middle cerebral artery occlusion; ATP, adenosine triphosphate.

endogenous small molecule metabolism. The results show that PNGL exerts a neuroprotective effect by reducing the activation of glial cells and rescuing the abnormal metabolism of small molecule in the early acute phase with MCAO rats, which is specifically reflected in the reduced abnormal accumulation of glucose and citric acid, increased ATP metabolism rate, augmented rate of the malate aspartate shuttle, increased antioxidants and maintained ionic homeostasis. Our approach offers critical insight into exploring new mechanisms for the PNGL treatment of cerebral ischemic/reperfusion injury.

\section{Acknowledgments}

We thank all people in Xiaoping Pu laboratory (National Key Research Laboratory of Natural and Biomimetic Drugs, Peking University) for assistance in experimental method and data processing.

Funding: This work was supported by the major projects of the National Natural Science Foundation of China (No.
81891012), The Drug Innovation Major Project (No. 2018ZX09711001-009), The National Key R\&D Plan (No. 2017YFC1702504), Central Public-Interest Scientific Institution Basal Research Fund (No. 2018PT35030), and The National Key R\&D Plan (No. 2018YFC1707408), The Drug Innovation Major Project (No. 2015ZX09101020).

\section{Footnote}

Reporting Checklist: The authors have completed the ARRIVE reporting checklist. Available at http://dx.doi. org/10.21037/atm-20-4898

Data Sharing Statement: Available at http://dx.doi. org/10.21037/atm-20-4898

Peer Review File: Available at http://dx.doi.org/10.21037/ atm-20-4898

Conflicts of Interest: All authors have completed the ICMJE 
uniform disclosure form (available at http://dx.doi. org/10.21037/atm-20-4898). The authors have no conflicts of interest to declare.

Ethical Statement: The authors are accountable for all aspects of the work in ensuring that questions related to the accuracy or integrity of any part of the work are appropriately investigated and resolved. The study was approved by the Institutional Animal Care and Use Committee of Chinese Academy of Medical Sciences and Peking Union Medical College (SYXK 2017-0020). All rats care and experimental procedures were reported in accordance with the Laboratory Animal Ethics Committee of the Institute of Medicinal Plant Development, Peking Union Medical College and complied with NIH Guidelines for the Care and Use of Laboratory Animals.

Open Access Statement: This is an Open Access article distributed in accordance with the Creative Commons Attribution-NonCommercial-NoDerivs 4.0 International License (CC BY-NC-ND 4.0), which permits the noncommercial replication and distribution of the article with the strict proviso that no changes or edits are made and the original work is properly cited (including links to both the formal publication through the relevant DOI and the license). See: https://creativecommons.org/licenses/by-nc-nd/4.0/.

\section{References}

1. George PM, Steinberg GK. Novel Stroke Therapeutics: Unraveling Stroke Pathophysiology and Its Impact on Clinical Treatments. Neuron 2015;87:297-309.

2. Minnerup J, Sutherland BA, Buchan AM, et al. Neuroprotection for stroke: current status and future perspectives. Int J Mol Sci 2012;13:11753-72.

3. Feigin VL, Forouzanfar MH, Krishnamurthi R, et al. Global and regional burden of stroke during 1990-2010: findings from the Global Burden of Disease Study 2010. Lancet 2014;383:245-54.

4. Bennett DA, Krishnamurthi RV, Barker-Collo S, et al. The global burden of ischemic stroke: findings of the GBD 2010 study. Glob Heart 2014;9:107-12.

5. Zhou Z, Lu J, Liu WW, et al. Advances in stroke pharmacology. Pharmacol Ther 2018;191:23-42.

6. Caprioli RM, Farmer TB, Gile J. Molecular imaging of biological samples: localization of peptides and proteins using MALDI-TOF MS. Anal Chem 1997;69:4751-60.

7. Liu H, Chen R, Wang J, et al. 1,5-Diaminonaphthalene hydrochloride assisted laser desorption/ionization mass spectrometry imaging of small molecules in tissues following focal cerebral ischemia. Anal Chem 2014;86:10114-21.

8. Tian F, Liu R, Fan C, et al. Effects of Thymoquinone on Small-Molecule Metabolites in a Rat Model of Cerebral Ischemia Reperfusion Injury Assessed using MALDI-MSI. Metabolites 2020;10:27.

9. Nilsson A, Fehniger TE, Gustavsson L, et al. Fine mapping the spatial distribution and concentration of unlabeled drugs within tissue micro-compartments using imaging mass spectrometry. PLoS One 2010;5:e11411.

10. Xu J, Zhang Z, Liu R, et al. Function of complement factor $\mathrm{H}$ and imaging of small molecules by MALDI-MSI in a methamphetamine behavioral sensitization model. Behav Brain Res 2019;364:233-44.

11. Chaurand P, Norris JL, Cornett DS, et al. New developments in profiling and imaging of proteins from tissue sections by MALDI mass spectrometry. J Proteome Res 2006;5:2889-900.

12. Clemis EJ, Smith DS, Camenzind AG, et al. Quantitation of spatially-localized proteins in tissue samples using MALDI-MRM imaging. Anal Chem 2012;84:3514-22 .

13. Cornett DS, Reyzer ML, Chaurand P, et al. MALDI imaging mass spectrometry: molecular snapshots of biochemical systems. Nat Methods 2007;4:828-33.

14. Oppenheimer SR, Wehr AY. Imaging mass spectrometry in drug discovery and development. Bioanalysis 2015;7:2609-10.

15. Li $W$, Liu $H$, Jiang $H$, et al. (S)-Oxiracetam is the Active Ingredient in Oxiracetam that Alleviates the Cognitive Impairment Induced by Chronic Cerebral Hypoperfusion in Rats. Sci Rep 2017;7:10052.

16. Leung AW, Mo ZX, Zheng YS. Reduction of cellular damage induced by cerebral ischemia in rats. Neurochem Res 1991;16:687-92.

17. Yao CL, Pan HQ, Wang H, et al. Global profiling combined with predicted metabolites screening for discovery of natural compounds: Characterization of ginsenosides in the leaves of Panax notoginseng as a case study. J Chromatogr A 2018;1538:34-44.

18. Fu JH, Li XZ, Shang XH, et al. Protective effects of saponines of stem and leaf of Panax notoginseng on acute myocardial ischemia in anaesthetic dogs. Zhongguo Zhong Yao Za Zhi 2006;31:62-5.

19. Chen ZH, Wang DC, Li HL, et al. Hemodynamic effects of san chi (Panax notoginseng) root, leaf, flower and saponins on anesthetized dogs. Yao Xue Xue Bao 
1983;18:818-22.

20. Liu XY, Wang S, Li CJ, et al. Dammarane-type saponins from the leaves of Panax notoginseng and their neuroprotective effects on damaged SH-SY5Y cells. Phytochemistry 2018;145:10-7.

21. Xie W, Zhu T, Dong X, et al. HMGB1-triggered inflammation inhibition of notoginseng leaf triterpenes against cerebral ischemia and reperfusion injury via MAPK and NF- $\kappa$ B signaling pathways. Biomolecules 2019;9:512.

22. Meng X, Wang M, Wang X, et al. Suppression of NADPH oxidase- and mitochondrion-derived superoxide by Notoginsenoside R1 protects against cerebral ischemiareperfusion injury through estrogen receptor-dependent activation of Akt/Nrf2 pathways. Free Radic Res 2014;48:823-38.

23. Wang Z, Huang Y, Cheng Y, et al. Endoplasmic reticulum stress-induced neuronal inflammatory response and apoptosis likely plays a key role in the development of diabetic encephalopathy. Oncotarget 2016;7:78455-72.

24. Liu RZ, Fan CX, Zhang ZL, et al. Effects of Dl-3-nbutylphthalide on Cerebral Ischemia Infarction in Rat Model by Mass Spectrometry Imaging. Int J Mol Sci 2017;18:2451.

25. Cao Y, Yang Y, Wu H, et al. Stem-leaf saponins from Panax notoginseng counteract aberrant autophagy and apoptosis in hippocampal neurons of mice with cognitive impairment induced by sleep deprivation. J Ginseng Res 2020;44:442-52.

26. Jeitner TM, Battaile K, Cooper AJ. Critical Evaluation of the Changes in Glutamine Synthetase Activity in Models of Cerebral Stroke. Neurochem Res 2015;40:2544-56.

27. Altinok O, Poggio JL, Stein DE, et al. Malate-aspartate

Cite this article as: Wang $\mathrm{L}, \mathrm{Zhu} \mathrm{T}, \mathrm{Xu} \mathrm{HB}, \mathrm{Pu} \mathrm{XP}$, Zhao X, Tian F, Ding T, Sun GB, Sun XB. Effects of notoginseng leaf triterpenes on small molecule metabolism after cerebral ischemia/reperfusion injury assessed using MALDIMS imaging. Ann Transl Med 2021;9(3):246. doi: 10.21037/atm20-4898 shuttle promotes 1-lactate oxidation in mitochondria. J

Cell Physiol 2020;235:2569-81.

28. Moffett JR, Ross B, Arun P, et al. N-Acetylaspartate in the CNS: from neurodiagnostics to neurobiology. Prog Neurobiol 2007;81:89-131.

29. Dimroth P, von Ballmoos C. ATP synthesis by decarboxylation phosphorylation. Results Probl Cell Differ 2008;45:153-84.

30. Barsotti C, Ipata PL. Metabolic regulation of ATP breakdown and of adenosine production in rat brain extracts. Int J Biochem Cell Biol 2004;36:2214-25.

31. Folbergrova J, Zhao Q, Katsura K, et al. N-tert-butylalpha-phenylnitrone improves recovery of brain energy state in rats following transient focal ischemia. Proc Natl Acad Sci U S A 1995;92:5057-61.

32. Sherif IO, Al-Shaalan NH. Vildagliptin Attenuates Hepatic Ischemia/Reperfusion Injury via the TLR4/ NF- $\kappa$ B Signaling Pathway. Oxid Med Cell Longev 2018;2018:3509091.

33. Szydlowska K, Tymianski M. Calcium, ischemia and excitotoxicity. Cell Calcium 2010;47:122-9.

34. Cross HR, Radda GK, Clarke K. The role of $\mathrm{Na}+\mathrm{K}+$ ATPase activity during low flow ischemia in preventing myocardial injury: a $31 \mathrm{P}, 23 \mathrm{Na}$ and $87 \mathrm{Rb} \mathrm{NMR}$ spectroscopic study. Magn Reson Med 1995;34:673-85.

35. Nagafuji T, Koide T, Takato M. Neurochemical correlates of selective neuronal loss following cerebral ischemia: role of decreased $\mathrm{Na}+\mathrm{K}(+)$-ATPase activity. Brain Res 1992;571:265-71.

36. Yang GY, Chen SF, Kinouchi H, et al. Edema, cation content, and ATPase activity after middle cerebral artery occlusion in rats. Stroke 1992;23:1331-6. 


\section{Supplementary}

A

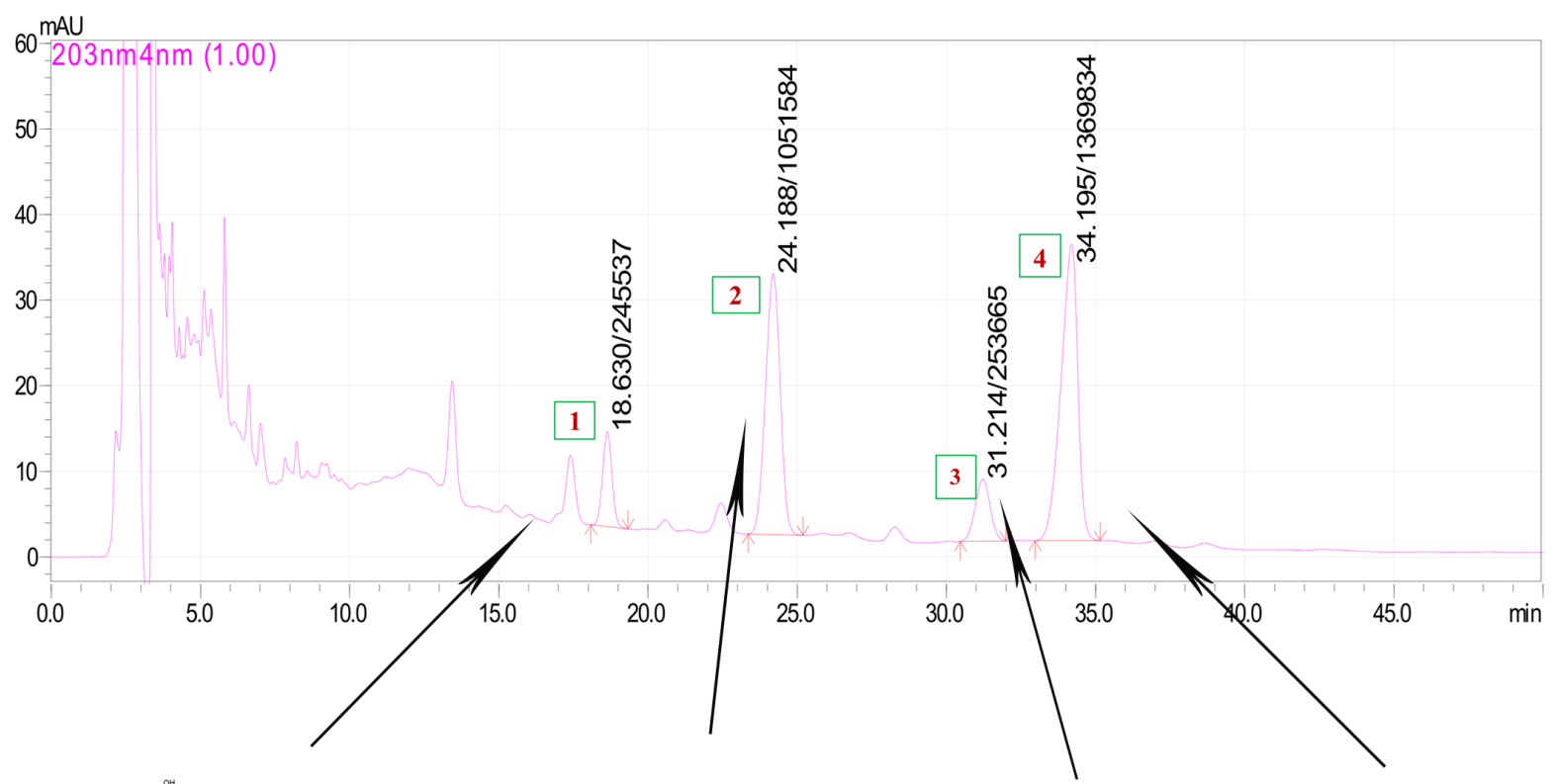

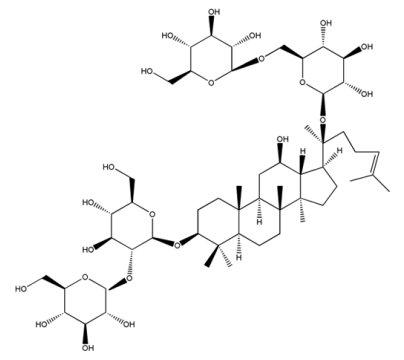

ginsenoside $\mathbf{R b} 1$

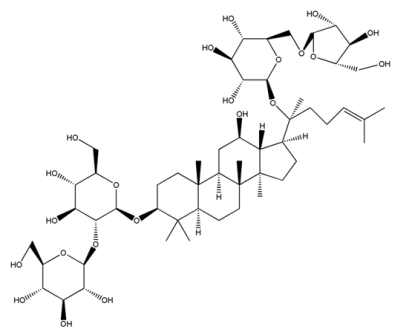

ginsenoside Rc

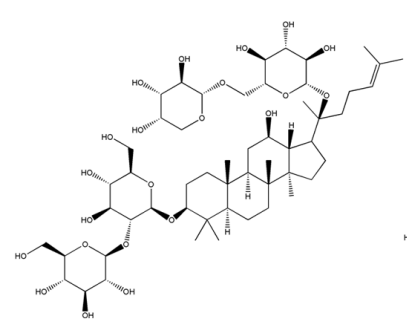

ginsenoside $\mathbf{R b} 2$

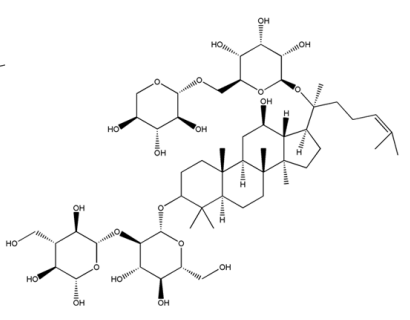

ginsenoside Rb3

Figure S1 The chromatograms and chemical components of PNGL detected by HPLC. (A) The chromatograms of PNGL samples. (B) Representative chemical component structures, primarily contains ginsenoside Rb1, ginsenoside Rc, ginsenoside Rb2, ginsenoside Rb3. PNGL, notoginseng leaf triterpenes; HPLC, high-performance liquid chromatography.

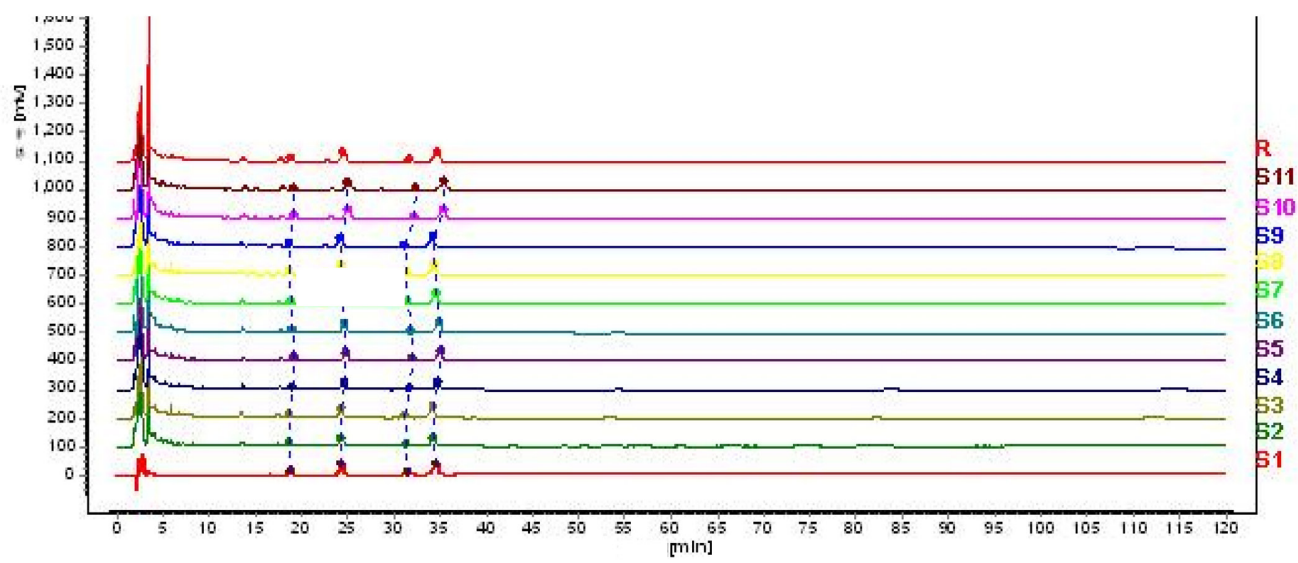

Figure S2 The chemical fingerprinting of PNGL detected by the HPLC. The chemical fingerprinting from elven batches of PNGL samples. PNGL, notoginseng leaf triterpenes; HPLC, high-performance liquid chromatography. 